\title{
The conformal supercurrents in diverse dimensions and conserved superconformal currents
}

Yegor Korovin, ${ }^{a}$ Sergei M. Kuzenko ${ }^{b}$ and Stefan Theisen ${ }^{a}$

${ }^{a}$ Max-Planck-Institut für Gravitationsphysik, Albert-Einstein-Institut, Am Mühlenberg 1, D-14476 Golm, Germany

${ }^{b}$ School of Physics M013, The University of Western Australia, 35 Stirling Highway, Crawley, W.A. 6009, Australia

E-mail: jegor.korovins@aei.mpg.de, sergei.kuzenko@uwa.edu.au, stefan.theisen@aei.mpg.de

ABSTRACT: Given a conserved and traceless energy-momentum tensor and a conformal Killing vector, one obtains a conserved current. We generalise this construction to superconformal theories in three, four, five and six dimensions with various amounts of supersymmetry by working in the appropriate superspaces.

KEYWORDS: Extended Supersymmetry, Superspaces, Supersymmetric Effective Theories ARXIV EPRINT: 1604.00488 


\section{Contents}

1 Introduction 1

2 Conformal Killing supervector fields 3

3 Superconformal theories in three dimensions 5

$\begin{array}{ll}3.1 \mathcal{N}=1 \text { superconformal symmetry } & 6\end{array}$

$\begin{array}{ll}3.2 \mathcal{N}=2 \text { superconformal symmetry } & 7\end{array}$

$3.3 \mathcal{N}=3$ superconformal symmetry 8

$3.4 \mathcal{N}=4$ superconformal symmetry $\quad 9$

4 Superconformal theories in four dimensions $\quad 10$

$\begin{array}{ll}4.1 \mathcal{N}=1 \text { superconformal symmetry } & 10\end{array}$

$\begin{array}{lll}4.2 \mathcal{N}=2 \text { superconformal symmetry } & 11\end{array}$

5 Superconformal theories in five and six dimensions 11

6 Non-conformal supercurrents, curved backgrounds $\quad 13$

$\begin{array}{ll}\text { A Conformal symmetries of curved superspace } & 15\end{array}$

\section{Introduction}

We consider conformal field theories in $d$-dimensional Minkowski space $\mathbb{M}^{d}$. A central feature of conformal field theories is the existence of a symmetric, traceless and conserved energy-momentum tensor $T^{a b}$

$$
T^{a b}=T^{b a}, \quad \eta_{a b} T^{a b}=0, \quad \partial_{b} T^{a b}=0,
$$

with $\eta_{a b}$ the Minkowski metric (of mostly plus signature). Let $\xi=\xi^{a} \partial_{a}$ be a conformal Killing vector field of $\mathbb{M}^{d}$,

$$
\partial_{a} \xi_{b}+\partial_{b} \xi_{a}=\frac{2}{d} \eta_{a b} \partial_{c} \xi^{c}
$$

As is well-known, for every conformal Killing vector $\xi$ one can construct a conserved current $V^{a}$ as

$$
V^{a}=T^{a b} \xi_{b}
$$

Conservation of $V^{a}$,

$$
\partial_{a} V^{a}=0
$$

is a consequence of the conservation and tracelessness of $T^{a b}$ (which hold on-shell). 
In this note, we generalise the above construction, $\left(T^{a b}, \xi^{a}\right) \rightarrow V^{a}$, to the case of superconformal field theories in diverse dimensions in a manifestly supersymmetric way. In the supersymmetric generalisation the traceless energy momentum tensor $T^{a b}$ will be embedded in the conformal supercurrent multiplet while the conserved conformal current $V^{a}$ will turn into the conserved superconformal current embedded in a supermultiplet.

A supersymmetric analogue of the energy-momentum tensor is the supercurrent multiplet introduced by Ferrara and Zumino [1] in the framework of four-dimensional (4D) $\mathcal{N}=1$ Poincaré supersymmetry. A supersymmetric extension of the notion of conserved current is the conserved current multiplet introduced by Ferrara, Wess and Zumino [2] in the 4D $\mathcal{N}=1$ super-Poincaré case. The concepts of supercurrent and conserved current multiplet also exist for different types of supersymmetry and in spacetimes of dimension $d \neq 4$. By definition, the supercurrent is a supermultiplet containing the energy-momentum tensor and the supersymmetry current(s), along with some additional components such as the $R$-symmetry current. In this note, we define the conserved current multiplet to be a supermultiplet containing a single conserved vector current (equivalently, a closed $(d-1)$-form), along with some other scalar and spinor components.

Unlike the energy-momentum tensor, the functional structure of the supercurrent and the corresponding conservation equation depend on the dimension and supersymmetry type. As an example we recall the $\mathcal{N}=1$ and $\mathcal{N}=2$ supersymmetric extensions of the conformal energy-momentum tensor (1.1) in four dimensions. The $\mathcal{N}=1$ conformal supercurrent [1] is a real vector superfield $J_{\alpha \dot{\alpha}}$ constrained by

$$
D^{\alpha} J_{\alpha \dot{\alpha}}=0 \quad \Longleftrightarrow \quad \bar{D}^{\dot{\alpha}} J_{\alpha \dot{\alpha}}=0 \text {. }
$$

The $\mathcal{N}=2$ conformal supercurrent [3] is a real scalar superfield $J$ constrained by

$$
D^{i j} J=0 \quad \Longleftrightarrow \quad \bar{D}^{i j} J=0
$$

where $D^{i j}=D^{\alpha i} D_{\alpha}^{j}=D^{j i}, \bar{D}^{i j}=\bar{D}_{\dot{\alpha}}^{i} \bar{D}^{j \dot{\alpha}}=\bar{D}^{j i}$. It is also pertinent to recall the $\mathcal{N}=1$ and $\mathcal{N}=2$ supersymmetric extensions of the conservation equation (1.4) in four dimensions. The $\mathcal{N}=1$ conserved current multiplet is described by a real linear superfield $L[2]$ constrained by

$$
D^{2} L=0 \quad \Longleftrightarrow \quad \bar{D}^{2} L=0
$$

In the $\mathcal{N}=2$ case, the conserved current multiplet is described by a real linear superfield $L^{i j}$ [4], which is defined to be a real SU(2) triplet $L^{i j}=L^{j i}, \overline{L^{i j}}=L_{i j}=\varepsilon_{i k} \varepsilon_{j l} L^{k l}$, constrained by

$$
D_{\alpha}^{(i} L^{j k)}=0 \quad \Longleftrightarrow \quad \bar{D}_{\dot{\alpha}}^{(i} L^{j k)}=0
$$

In order to introduce a supersymmetric analogue of the construction, $\left(T^{a b}, \xi^{a}\right) \rightarrow V^{a}$, we also need a supersymmetric counterpart of the notion of conformal Killing vector. It was originally given by Sohnius [5] in the case of $4 \mathrm{D} \mathcal{N}$-extended Poincaré supersymmetry and further developed in four [6-12] and other [13-17] dimensions.

In the next section we review the definition of conformal Killing supervector fields. This is further elaborated in appendix A. Sections 3 to 5 are devoted to superconformal theories 
in three, four, five and six dimensions, respectively. In each of these sections we first review known results about the superconformal currents. We also state the general properties of conserved current multiplets and their conservation laws. We then construct these conserved currents in terms of the supercurrents and the conformal Killing supervector fields, generalising eq. (1.3). Section 6 discusses modifications that occur for non-conformal supercurrents and in the case of curved backgrounds.

\section{Conformal Killing supervector fields}

According to Nahm's classification [18], superconformal algebras exist in spacetime dimensions $d \leq 6$. In superspace, the superconformal transformations are generated by the so-called conformal Killing supervector fields. The latter can be defined in several different but equivalent ways. We first recall the least orthodox but, probably, most useful definition. We then present the more standard definition.

Let $\mathbb{M}^{d \mid \delta}$ be a Minkowski superspace with $d \leq 6$ spacetime dimensions and $\delta$ fermionic dimensions. We denote by $z^{A}=\left(x^{a}, \theta^{\hat{\alpha}}\right)$ the bosonic $\left(x^{a}\right)$ and fermionic $\left(\theta^{\hat{\alpha}}\right)$ coordinates for $\mathbb{M}^{d \mid \delta}$. The index $\hat{\alpha}$ of the Grassmann variable is, in general, composite; it is comprised of a spinor index $\boldsymbol{\alpha}$ and an $R$-symmetry index $I$. The superspace covariant derivatives are $D_{A}=\left(\partial_{a}, D_{\hat{\alpha}}\right)=e_{A}{ }^{M} \partial_{M}$ such that $\left\{D_{\hat{\alpha}}, D_{\hat{\beta}}\right\}=T_{\hat{\alpha} \hat{\beta}}{ }^{c} \partial_{c}$ and $\left[D_{\hat{\alpha}}, \partial_{b}\right]=0$, where $T_{\hat{\alpha} \hat{\beta}}{ }^{c}$ is the flat-superspace torsion tensor, which is constant.

A real even supervector field ${ }^{1}$ over $\mathbb{M}^{d \mid \delta}, \xi=\bar{\xi}=\xi^{A} D_{A}=\xi^{a} \partial_{a}+\xi^{\hat{\alpha}} D_{\hat{\alpha}}$, is said to be conformal Killing if the following condition holds

$$
\left[\xi, D_{\hat{\alpha}}\right] \propto D_{\hat{\beta}} \quad \Longleftrightarrow \quad\left[\xi, D_{\hat{\alpha}}\right]=-\left(D_{\hat{\alpha}} \xi^{\hat{\beta}}\right) D_{\hat{\beta}} .
$$

This condition implies that the only independent components of $\xi$ are the vector ones, $\xi^{a}$. The set of all conformal Killing supervector fields forms a superalgebra (with respect to the standard Lie bracket) which is isomorphic to the superconformal algebra.

The above definition was used to introduce the conformal Killing supervector fields in the 5D [16] and 3D $\mathcal{N}$-extended [17] cases. However, it differs somewhat from that used in [12] in the $4 \mathrm{D} \mathcal{N}$-extended case, as a generalisation of the $4 \mathrm{D} \mathcal{N}=1$ analysis in [9]. Let us show that the definition used in [12] follows from the one above.

The coordinates of $4 \mathrm{D} \mathcal{N}$-extended Minkowski superspace $\mathbb{M}^{4 \mid 4 \mathcal{N}}$ are $z^{A}=\left(x^{a}, \theta_{i}^{\alpha}, \bar{\theta}_{\dot{\alpha}}^{i}\right)$, where $\alpha$ and $\dot{\alpha}$ are two-component spinor indices, and $i=1, \ldots, \mathcal{N}$ is an $R$-symmetry index. $^{2}$ The spinor covariant derivatives obey the anti-commutation relations

$$
\left\{D_{\alpha}^{i}, D_{\beta}^{j}\right\}=0, \quad\left\{\bar{D}_{\dot{\alpha} i}, \bar{D}_{\dot{\beta} j}\right\}=0, \quad\left\{D_{\alpha}^{i}, \bar{D}_{\dot{\beta} j}\right\}=-2 \mathrm{i} \delta_{j}^{i} \partial_{\alpha \dot{\beta}},
$$

where $\partial_{\alpha \dot{\beta}}=\left(\sigma^{c}\right)_{\alpha \dot{\beta}} \partial_{c}$. Given a supervector field $\xi=\xi^{a} \partial_{a}+\xi_{i}^{\alpha} D_{\alpha}^{i}+\bar{\xi}_{\dot{\alpha}}^{i} \bar{D}_{i}^{\dot{\alpha}}$, the condition (2.1) implies

$$
\bar{D}_{\dot{\alpha} i} \xi^{\dot{\beta} \beta}=4 \mathrm{i} \delta_{\dot{\alpha}}^{\dot{\beta}} \xi_{i}^{\beta}
$$

\footnotetext{
${ }^{1} \mathrm{~A}$ supervector field $\xi$ is real and even if $\xi \Phi$ is real and even for every real bosonic superfield $\Phi$. In what follows, all supervector fields will be real and even.

${ }^{2}$ Our 4 D notation and conventions follow $[9,19]$.
} 
Making use of this result, a short calculation gives

$$
\bar{D}_{\dot{\alpha} i} \bar{D}_{\dot{\beta} j} \xi^{\dot{\gamma} \gamma}=0 \quad \Longleftrightarrow \quad \bar{D}_{\dot{\alpha} i} \xi_{j}^{\beta}=0
$$

Equation (2.1) is thus equivalent to

$$
\left[\xi, D_{\alpha}^{i}\right] \propto D_{\beta}^{j},
$$

which is the definition of the conformal Killing supervector fields used in [12].

Eq. (2.3) is equivalent to the relations

$$
\begin{aligned}
& \xi_{i}^{\alpha}=-\frac{\mathrm{i}}{8} \bar{D}_{\dot{\alpha} i} \xi^{\dot{\alpha} \alpha} \Longleftrightarrow \quad \bar{\xi}_{\dot{\alpha}}^{i}=-\frac{\mathrm{i}}{8} D^{\alpha i} \xi_{\alpha \dot{\alpha}}, \\
& D_{(\alpha}^{i} \xi_{\beta) \dot{\beta}}=0 \quad \Longleftrightarrow \quad \bar{D}_{i}^{(\dot{\alpha}} \xi^{\dot{\beta}) \beta}=0 .
\end{aligned}
$$

Relations (2.6a) express the spinor components of $\xi^{A}$ in terms of the vector one. Eq. (2.6b) is the $4 \mathrm{D} \mathcal{N}$-extended superconformal Killing equation, which is a supersymmetric counterpart of (1.2). In conjunction with the definition (2.6a), it proves to contain all the information about the conformal Killing supervector fields. An obvious consequence of (2.6b) is that the vector superfield parameter $\xi_{\beta \dot{\beta}}=\left(\sigma_{a}\right)_{\beta \dot{\beta}} \xi^{a}(x, \theta, \bar{\theta})$ obeys the equation

$$
\partial_{\left(\alpha \left(\dot{\alpha} \xi_{\beta) \dot{\beta})}\right.\right.}=0 \text {. }
$$

Switching off the Grassmann variables gives the vector field $\xi^{a}\left|:=\xi^{a}(x, \theta, \bar{\theta})\right|_{\theta=\bar{\theta}=0}$, which is an ordinary conformal Killing vector field. Indeed, (2.7) coincides with the $d=4$ conformal Killing equation (1.2) rewritten in the two-component spinor notation.

The traditional definition of superconformal transformations in superspace was originally given by Sohnius [5] in the $4 \mathrm{D} \mathcal{N}$-extended case. ${ }^{3}$ Park used this definition to introduce the superconformal transformations in the $6 \mathrm{D} \mathcal{N}=(p, 0)$ and $\mathcal{N}=(0, q)[14]$ and $3 \mathrm{D}$ $\mathcal{N}$-extended [15] Minkowski superspaces. According to this definition, an infinitesimal coordinate transformation $\delta z^{A}=\xi^{A}(z)$ generated by a supervector field $\xi^{A}$ on $\mathbb{M}^{d \mid \delta}$, is called superconformal if it at most scales the supersymmetric interval $\mathrm{d} s^{2}=\eta_{a b} e^{a} e^{b}$. Here the supersymmetric one-forms $[20,21] e^{A}=\mathrm{d} z^{M} e_{M}{ }^{A}$ constitute the dual basis for $D_{A}$ defined by $\mathrm{d}=\mathrm{d} z^{A} \partial_{A}=e^{A} D_{A}$. Using this definition, Park showed [14] that in six dimensions superconformal transformations and hence superconformal algebras exist only for the supersymmetry types $\mathcal{N}=(p, 0)$ and $\mathcal{N}=(0, q)$. While one can define $\mathcal{N}=(p, q)$ Poincaré supersymmetry for any non-negative integers $p$ and $q$, in the mixed case with $p, q \neq 0$, the most general conformal Killing supervector field describes only super-Poincaré, $R$-symmetry and scale transformations. Analogous considerations may be used to show that in five dimensions, where one can introduce $\mathcal{N}$-extended Poincaré supersymmetry (with $8 \mathcal{N}$ supercharges), superconformal algebras exists only for $\mathcal{N}=1$; see [22] for a recent derivation.

It is an instructive exercise to show that invariance of $\mathrm{d} s^{2}$ leads to (2.1) which in turn allows to express $\xi^{\hat{a}}$ in terms of $\xi^{a}$ which itself satisfies the conformal Killing equation. Equivalence of the two definitions of conformal Killing supervector fields may also be established using a more general (third) definition, which is reviewed in appendix A.

\footnotetext{
${ }^{3}$ Sohnius simply generalised the standard definition of infinitesimal conformal transformations of $\mathbb{M}^{d}$ as those transformations which at most scale the interval $\mathrm{d} s^{2}=\eta_{a b} \mathrm{~d} x^{a} \mathrm{~d} x^{b}$, where $\eta_{a b}$ is the Minkowski metric.
} 


\section{Superconformal theories in three dimensions}

In this section we consider superconformal field theories in three dimensions. The $3 \mathrm{D} \mathcal{N}$ extended conformal supercurrents have been described in [23] using the conformal superspace formulation for $\mathcal{N}$-extended conformal supergravity, which was developed in [24]. In the locally supersymmetric case, the supercurrent of a superconformal field theory coupled to conformal supergravity is characterised by the same superfield type and the differential constraints as the super-Cotton tensor, which is the only independent curvature tensor of $\mathcal{N}$-extended conformal superspace [24]. Upon freezing the conformal superspace to $\mathcal{N}$-extended Minkowski superspace $\mathbb{M}^{3 \mid 2 \mathcal{N}}$, we end up with the conformal supercurrents which were discussed in detail in $[25,26]$ and which we are going to review. Here we parametrise $\mathbb{M}^{3 \mid 2 \mathcal{N}}$ by real Cartesian coordinates $z^{A}=\left(x^{a}, \theta_{I}^{\alpha}\right)$, where $I=1, \ldots, \mathcal{N}$. The spinor covariant derivatives $D_{\alpha}^{I}$ on $\mathbb{M}^{3 \mid 2 \mathcal{N}}$ satisfy the anti-commutation relation $\left\{D_{\alpha}^{I}, D_{\beta}^{J}\right\}=2 \mathrm{i} \delta^{I J} \gamma_{\alpha \beta}^{m} \partial_{m}$; see [17] for further details.

For every $\mathcal{N}=1,2 \ldots$, the conformal supercurrent is a primary real tensor superfield in the sense of the superconformal transformation law (5.1) in [25]. The conformal supercurrents $^{4}$ for $\mathcal{N} \leq 4$ are specified by the following properties:

\begin{tabular}{|c|c|c|c|}
\hline SUSY Type & Supercurrent & Dimension & Conservation Equation \\
\hline $\mathcal{N}=1$ & $J_{\alpha \beta \gamma}$ & $5 / 2$ & $D^{\alpha} J_{\alpha \beta \gamma}=0$ \\
\hline $\mathcal{N}=2$ & $J_{\alpha \beta}$ & 2 & $D^{I \alpha} J_{\alpha \beta}=0$ \\
\hline $\mathcal{N}=3$ & $J_{\alpha}$ & $3 / 2$ & $D^{I \alpha} J_{\alpha}=0$ \\
\hline $\mathcal{N}=4$ & $J$ & 1 & $\left(D^{I \alpha} D_{\alpha}^{J}-\frac{1}{4} \delta^{I J} D^{L \alpha} D_{\alpha}^{L}\right) J=0$ \\
\hline
\end{tabular}

For $\mathcal{N}>4$, the conformal supercurrent is a completely antisymmetric dimension-1 superfield, $J^{I J K L}=J^{[I J K L]}$, subject to the conservation equation

$$
D_{\alpha}^{I} J^{J K L P}=D_{\alpha}^{[I} J^{J K L P]}-\frac{4}{\mathcal{N}-3} D_{\alpha}^{Q} J^{Q[J K L} \delta^{P] I} .
$$

In the $\mathcal{N}=4$ case, this equation is identically satisfied for $J^{I J K L}=\varepsilon^{I J K L} J$. That is why the $\mathcal{N}=4$ supercurrent $J$ obeys the second-order conservation equation given in (3.1).

In three dimensions, one may think of a conserved current $V^{a}$, eq. (1.4), as the Hodge dual of the gauge-invariant field strength $F=\mathrm{d} A$ of a gauge one-form $A$. For this reason an $\mathcal{N}$-extended conserved current multiplet may be characterised by the same superfield type and the differential constraints as the field strength of an $\mathcal{N}$-extended Abelian vector multiplet [29-33]. The conserved current multiplets with $\mathcal{N} \leq 4$ were reviewed in [25, 26]. In the $\mathcal{N}=1$ case, the conserved current multiplet is a real spinor superfield $L^{\alpha}$ constrained by

$$
D^{\alpha} L_{\alpha}=0 \text {. }
$$

For $\mathcal{N}>1$, it is a real antisymmetric superfield, $L^{I J}=-L^{J I}$, constrained by

$$
D_{\alpha}^{I} L^{J K}=D_{\alpha}^{[I} L^{J K]}-\frac{2}{\mathcal{N}-1} D_{\alpha}^{L} L^{L[J} \delta^{K] I}
$$

\footnotetext{
${ }^{4}$ The $\mathcal{N}=2$ (non-)conformal supercurrents were studied in $[27,28]$.
} 
This equation is identically satisfied in the $\mathcal{N}=2$ case for which $L^{I J}=\varepsilon^{I J} L$. For $\mathcal{N}=2$ the conserved current multiplet obeys instead the conservation equation

$$
\left(D^{\alpha I} D_{\alpha}^{J}-\frac{1}{2} \delta^{I J} D^{K \alpha} D_{\alpha}^{K}\right) L=0 .
$$

When $\mathcal{N}=3$, it is more convenient to work with the Hodge dual of $L^{I J}$, which is $L^{I}=\frac{1}{2} \varepsilon^{I J K} L^{J K}$. The latter obeys the constraint

$$
D_{\alpha}^{(I} L^{J)}-\frac{1}{3} \delta^{I J} D_{\alpha}^{K} L^{K}=0,
$$

which is equivalent to (3.4) with $\mathcal{N}=3$.

The $\mathcal{N}=4$ case is very special. Given an $\mathcal{N}=4$ conserved current multiplet $L^{I J}$, it can be uniquely represented as $L^{I J}=L_{+}^{I J}+L_{-}^{I J}$, where $L_{+}^{I J}$ and $L_{-}^{I J}$ are self-dual and anti-self-dual, respectively,

$$
\frac{1}{2} \varepsilon^{I J K L} L_{ \pm}^{K L}= \pm L_{ \pm}^{I J}
$$

It turns out that each of $L_{+}^{I J}$ and $L_{-}^{I J}$ obeys the conservation equation (3.4) with $\mathcal{N}=4$. Thus there are two inequivalent current multiplets in the $\mathcal{N}=4$ case. This is in accord with the fact that the $\mathcal{N}=4 R$-symmetry group factorises, due to the isomorphism $\mathrm{SO}(4) \cong$ $\left(\mathrm{SU}(2)_{\mathrm{L}} \times \mathrm{SU}(2)_{\mathrm{R}}\right) / \mathbb{Z}_{2}$.

For $\mathcal{N}>4$, it turns out that the off-shell multiplet defined by (3.4) possesses more than one conserved current at the component level. Moreover, it also contains higher spin conserved currents for $\mathcal{N}>5[34,35]$. Therefore, there is no conserved current multiplet for $\mathcal{N}>4$ in the sense of the definition given in section 1 .

In the cases $\mathcal{N}=2,3,4$, it is often convenient to switch from the real basis for the Grassmann variables $\theta_{I}^{\alpha}$ to a complex one in accordance with the rules described in [17]. Schematically, this amounts to replacements: (i) $D_{\alpha}^{I} \rightarrow\left(D_{\alpha}, \bar{D}_{\alpha}\right)$ for $\mathcal{N}=2$; (ii) $D_{\alpha}^{I} \rightarrow$ $D_{\alpha}^{i j}=D_{\alpha}^{j i}$ for $\mathcal{N}=3$, where $i, j=1,2$; (ii) $D_{\alpha}^{I} \rightarrow D_{\alpha}^{i \bar{i}}$ for $\mathcal{N}=4$, where $i, \bar{i}=1,2$. We will use such types of parametrisation in the remainder of this section, where we discuss the conserved currents for $\mathcal{N}=1,2,3,4$ in turn.

\section{$3.1 \mathcal{N}=1$ superconformal symmetry}

Any supervector field $\xi$ on $\mathcal{N}=1$ Minkowski superspace $\mathbb{M}^{3 \mid 2}$ has the expansion

$$
\xi=\xi^{A} D_{A}=\xi^{a} \partial_{a}+\xi^{\alpha} D_{\alpha},
$$

with the vector $\xi^{a}$ and spinor $\xi^{\alpha}$ components being real. Requiring $\xi$ to be conformal Killing, eq. (2.1), leads to the following conditions:

$$
\begin{aligned}
\xi^{\alpha} & =\frac{\mathrm{i}}{6} D_{\beta} \xi^{\beta \alpha}, \\
D_{(\gamma} \xi_{\alpha \beta)} & =0
\end{aligned}
$$

of which $(3.9 \mathrm{~b})$ is the $\mathcal{N}=1$ superconformal Killing equation. With the help of the conformal supercurrent $J^{\alpha \beta \gamma}$, which satisfies the conservation equation

$$
D_{\gamma} J^{\alpha \beta \gamma}=0 \quad \Longrightarrow \quad \partial_{\beta \gamma} J^{\alpha \beta \gamma}=0 \text {. }
$$


we construct the following spinor superfield:

$$
L^{\alpha}=-\frac{1}{2} \xi_{\beta \gamma} J^{\alpha \beta \gamma} .
$$

It follows from (3.9b) and (3.10) that $L^{\alpha}$ obeys equation (3.3), which defines a conserved current multiplet.

A few words are in order about the component structure of $J^{\alpha \beta \gamma}$ and $L^{\alpha}$. As follows from (3.9b), the supercurrent has two independent real component fields, which are:

$$
S^{\alpha \beta \gamma}:=J^{\alpha \beta \gamma}\left|, \quad T^{\alpha \beta \gamma \delta}:=D^{\delta} J^{\alpha \beta \gamma}\right|=T^{(\alpha \beta \gamma \delta)},
$$

where the bar-projection means, as usual, that the Grassmann variables must be switched off. Here $S^{\alpha \beta \gamma}$ is the supersymmetry current, and $T^{\alpha \beta \gamma \delta}$ the energy-momentum tensor. Both currents are conserved,

$$
\partial_{\alpha \beta} S^{\alpha \beta \gamma}=0, \quad \partial_{\alpha \beta} T^{\alpha \beta \gamma \delta}=0 .
$$

Switching to the three-vector notation, $T^{\alpha \beta \gamma \delta} \rightarrow T^{a b}=\frac{1}{4}\left(\gamma^{a}\right)_{\alpha \beta}\left(g^{b}\right)_{\gamma \delta} T^{\alpha \beta \gamma \delta}=T^{b a}$ and $S^{\alpha \beta \gamma} \rightarrow S^{a \gamma}=-\frac{1}{2}\left(\gamma^{a}\right)_{\alpha \beta} S^{\alpha \beta \gamma}$, the energy-momentum is automatically traceless, $\eta_{a b} T^{a b}=$ 0 , and so is the supersymmetry current, $\gamma_{a} S^{a}=0$.

Given a conserved current multiplet $L^{\alpha}$ constrained by (3.3), it has two independent real component fields, which can be chosen as

$$
\lambda^{\alpha}:=L^{\alpha}\left|, \quad V^{\alpha \beta}:=D^{\beta} L^{\alpha}\right|=V^{\beta \alpha} .
$$

The vector field is conserved,

$$
\partial_{\alpha \beta} V^{\alpha \beta}=0 .
$$

To compute the conserved current contained in (3.11), one needs the explicit expression for an arbitrary $\mathcal{N}=1$ conformal Killing supervector field. The most general $\mathcal{N}$-extended conformal Killing supervector field is given by eq. (4.4) in [25].

\section{$3.2 \mathcal{N}=2$ superconformal symmetry}

Any supervector field $\xi$ on $\mathcal{N}=2$ Minkowski superspace $\mathbb{M}^{3 \mid 4}$ has the form

$$
\xi=\xi^{A} D_{A}=\xi^{a} \partial_{a}+\xi^{\alpha} D_{\alpha}+\bar{\xi}_{\alpha} \bar{D}^{\alpha},
$$

where $\xi^{a}$ is real, and $\bar{\xi}^{\alpha}$ is the complex conjugate of $\xi^{\alpha}$. Requiring $\xi$ to be conformal Killing, eq. (2.1), gives

$$
\begin{aligned}
\xi^{\alpha} & =-\frac{\mathrm{i}}{6} \bar{D}_{\beta} \xi^{\beta \alpha}, \\
D_{(\gamma} \xi_{\alpha \beta)} & =\bar{D}_{(\gamma} \xi_{\alpha \beta)}=0 \quad \Longrightarrow \quad D^{2} \xi_{\alpha \beta}=\bar{D}^{2} \xi_{\alpha \beta}=0 .
\end{aligned}
$$

Here (3.17b) is the $\mathcal{N}=2$ superconformal Killing equation. Together with the $\mathcal{N}=2$ conformal supercurrent $J^{\alpha \beta}$, which satisfies

$$
D_{\beta} J^{\alpha \beta}=\bar{D}_{\beta} J^{\alpha \beta}=0 \quad \Longrightarrow \quad \partial_{\alpha \beta} J^{\alpha \beta}=0,
$$


we construct the scalar superfield

$$
L=-\frac{1}{2} \xi_{\alpha \beta} J^{\alpha \beta}=\xi_{a} J^{a} .
$$

It follows from (3.17b) and (3.18) that $L$ is a linear superfield,

$$
D^{2} L=\bar{D}^{2} L=0
$$

and therefore $L$ contains a conserved current.

Because of the constraints (3.18), the supercurrent has four independent component fields, which are

$$
j^{\alpha \beta}:=J^{\alpha \beta}\left|, \quad S^{\alpha \beta \gamma}:=D^{\gamma} J^{\alpha \beta}\right|=S^{(\alpha \beta \gamma)}, \quad T^{\alpha \beta \gamma \delta}:=\left[D^{(\gamma}, \bar{D}^{\delta}\right] J^{\alpha \beta)} \mid,
$$

as well as $\bar{S}^{\alpha \beta \gamma}$, the complex conjugate of $S^{\alpha \beta \gamma}$. Here $j^{\alpha \beta}$ is the $R$-symmetry current, $S^{\alpha \beta \gamma}$ and $\bar{S}^{\alpha \beta \gamma}$ the supersymmetry currents, and $T^{\alpha \beta \gamma \delta}$ the energy-momentum tensor. All these currents are conserved, as a consequence of the constraints (3.18).

Given a conserved current multiplet $L=\bar{L}$ constrained by (3.20), it has five independent components, which can be identified with

$$
l:=L\left|, \quad \lambda^{\alpha}:=D^{\alpha} L\right| \quad U:=\mathrm{i} D^{\alpha} \bar{D}_{\alpha} L\left|, \quad V^{\alpha \beta}:=\left[D^{(\alpha}, \bar{D}^{\beta)}\right] L\right|,
$$

as well as $\bar{\lambda}^{\alpha}$, the complex conjugate of $\lambda^{\alpha}$. The vector field is conserved, $\partial_{\alpha \beta} V^{\alpha \beta}=0$, as a consequence of the identity

$$
\left[D^{2}, \bar{D}^{2}\right]=-4 \mathrm{i} \partial_{\alpha \beta}\left[D^{\alpha}, \bar{D}^{\beta}\right]
$$

To compute the conserved current contained in (3.19), one has to make use of the explicit expression for the most general $\mathcal{N}=$ conformal Killing supervector field given in [25].

\section{$3.3 \mathcal{N}=3$ superconformal symmetry}

Any supervector field $\xi$ on $\mathcal{N}=3$ Minkowski superspace $\mathbb{M}^{3 \mid 6}$ has the form

$$
\xi=\xi^{A} D_{A}=\xi^{a} \partial_{a}+\xi_{i j}^{\alpha} D_{\alpha}^{i j}, \quad \xi_{i j}^{\alpha}=\xi_{j i}^{\alpha},
$$

where $i, j$ are $\mathrm{SU}(2) R$-symmetry indices. Requiring $\xi$ to be conformal Killing, eq. (2.1), and making use of the anti-commutation relation $\left\{D_{\alpha}^{i j}, D_{\beta}^{k l}\right\}=-2 \mathrm{i} \varepsilon^{i(k} \varepsilon^{l) j} \partial_{\alpha \beta}$, we deduce that

$$
\begin{aligned}
\xi_{\alpha}^{i j} & =-\frac{\mathrm{i}}{6} D^{\beta i j} \xi_{\alpha \beta}, \\
D_{(\alpha}^{i j} \xi_{\beta \gamma)} & =0 .
\end{aligned}
$$

Here (3.25b) is the $\mathcal{N}=3$ superconformal Killing equation. An important consequence one may derive from (3.25) is the identity

$$
D_{\alpha}^{(i j} \xi_{\beta}^{k l)}=0
$$


The $\mathcal{N}=3$ conformal supercurrent $J^{\alpha}$ satisfies

$$
D_{\alpha}^{i j} J^{\alpha}=0
$$

Let us define a real SU(2) triplet $L^{i j}=L^{j i}$ associated with $J^{\alpha}$ and $\xi^{A}$ by the rule:

$$
L^{i j}=\mathrm{i} \xi_{\alpha}^{i j} J^{\alpha}+\frac{1}{4} \xi^{\alpha \beta} D_{\alpha}^{i j} J_{\beta} .
$$

The properties of $J^{\alpha}$ and $\xi^{A}$ imply that $L^{i j}$ is a linear multiplet,

$$
D_{\alpha}^{(i j} L^{k l)}=0,
$$

and therefore $L^{i j}$ contains a conserved current.

Here we do not discuss the component content of $J^{\alpha}$ and $L^{i j}$. It can be readily determined, e.g., by making use of the $\mathcal{N}=3 \rightarrow \mathcal{N}=2$ superfield reduction of the $\mathcal{N}=3$ supercurrent and conserved current multiplets described in [25]. We only point out that the conserved current, which is contained in $L^{i j}$, is given by

$$
V_{\alpha \beta}=\mathrm{i} \varepsilon_{k l} D_{\alpha}^{i k} D_{\beta}^{j l} L_{i j} \mid=V_{\beta \alpha} .
$$

\section{$3.4 \mathcal{N}=4$ superconformal symmetry}

Given an $\mathcal{N}=4$ conformal Killing supervector field

$$
\xi=\xi^{A} D_{A}=\xi^{a} \partial_{a}+\xi_{i \bar{i}}^{\alpha} D_{\alpha}^{i \bar{i}},
$$

it follow from (2.1) that

$$
\begin{aligned}
\xi^{\alpha i \bar{i}} & =\frac{\mathrm{i}}{6} D_{\beta}^{i \bar{i}} \xi^{\beta \alpha}, \\
D_{(\alpha}^{i \bar{i}} \xi_{\beta \gamma)} & =0
\end{aligned}
$$

Here $D_{\alpha}^{i \bar{i}}$ is the $\mathcal{N}=4$ spinor covariant derivative defined as in [17], with the two-component indices $i$ and $\bar{i}$ corresponding to the left and right subgroups of the $R$-symmetry group $\mathrm{SU}(2)_{\mathrm{L}} \times \mathrm{SU}(2)_{\mathrm{R}}$, respectively. Eq. (3.32b) is the $\mathcal{N}=3$ superconformal Killing equation.

The $\mathcal{N}=4$ conformal supercurrent $J$ satisfies the conservation equation

$$
\varepsilon^{\alpha \beta} D_{\alpha}^{(i(\bar{i}} D_{\beta}^{j) \bar{j})} J=0 .
$$

Associated with $\xi^{A}$ and $J$ is a left $\mathrm{SU}(2)$ triplet $L^{i j}=L^{j i}$ defined by

$$
L^{i j}=\frac{\mathrm{i}}{4} \xi^{\alpha \beta} D_{\alpha}^{i \bar{k}} D_{\beta \bar{k}}^{j} J+\xi^{\alpha(i \bar{k}} D_{\alpha \bar{k}}^{j)} J+\Lambda^{i j} J,
$$

where we have introduced [17]

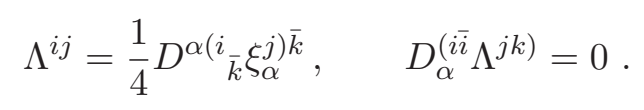

The properties of $\xi^{A}$ and $J$ imply that $L^{i j}$ is a left linear multiplet,

$$
D_{\alpha}^{(i \bar{i}} L^{j k)}=0
$$


and therefore $L^{i j}$ contains a conserved current.

In complete analogy with $L^{i j}$, one can also introduce a right SU $(2)$ triplet $L^{\bar{i} \bar{j}}=L^{\bar{j} i}$; it also contains a conserved current.

Here we do not discuss the component content of $J$ and $L^{i j}$. It can be readily determined, e.g., by making use of the $\mathcal{N}=4 \rightarrow \mathcal{N}=3$ superfield reduction of the $\mathcal{N}=4$ supercurrent and conserved current multiplets described in $[25,26]$. We only point out that the conserved current, which is contained in $L^{i j}$, is given by

$$
V_{\alpha \beta}=\mathrm{i} \varepsilon_{\bar{i} \bar{j}} D_{\alpha}^{i \bar{i}} D_{\beta}^{j \bar{j}} L_{i j} \mid=V_{\beta \alpha} .
$$

\section{Superconformal theories in four dimensions}

In four dimensions, we consider only the $\mathcal{N}=1$ and $\mathcal{N}=2$ superconformal theories, because these cases allow the existence of conserved current multiplets without higher spin fields [37].

\section{$4.1 \mathcal{N}=1$ superconformal symmetry}

Consider an arbitrary $\mathcal{N}=1$ conformal Killing supervector field,

$$
\xi=\xi^{a} \partial_{a}+\xi^{\alpha} D_{\alpha}+\bar{\xi}_{\dot{\alpha}} \bar{D}^{\dot{\alpha}}
$$

Its components are constrained according to (2.6). Let $J_{\alpha \dot{\alpha}}$ be the $\mathcal{N}=1$ conformal supercurrent. It is a primary real vector superfield of dimension +3 , as discussed, e.g., in [36]. The supercurrent conservation equation is given by eq. (1.5). Then the real scalar

$$
L=-\frac{1}{2} \xi^{\dot{\alpha} \alpha} J_{\alpha \dot{\alpha}}
$$

is a conserved current multiplet,

$$
D^{2} L=0 \quad \Longleftrightarrow \quad \bar{D}^{2} L=0
$$

It follows from (1.5) that the conformal supercurrent has four independent components, which can be chosen as follows:

$$
j_{\alpha \dot{\alpha}}:=J_{\alpha \dot{\alpha}}\left|, \quad S_{\alpha \beta \dot{\alpha}}:=D_{\beta} J_{\alpha \dot{\alpha}}\right|=S_{(\alpha \beta) \dot{\alpha}}, \quad T_{\alpha \beta \dot{\alpha} \dot{\beta}}:=\left[D_{(\beta}, \bar{D}_{(\dot{\beta}}\right] J_{\alpha) \dot{\alpha})} \mid,
$$

as well as the complex conjugate of $S_{\alpha \beta \dot{\alpha}}, \bar{S}_{\alpha \dot{\alpha} \dot{\beta}}$. Here $j_{\alpha \dot{\alpha}}$ is the $R$-symmetry current, $S_{\alpha \beta \dot{\alpha}}$ and $\bar{S}_{\alpha \dot{\alpha} \dot{\beta}}$ the supersymmetry currents, and $T_{\alpha \beta \dot{\alpha} \dot{\beta}}=T_{(\alpha \beta)(\dot{\alpha} \dot{\beta})}$ the energy-momentum tensor. ${ }^{5}$ All these currents are conserved,

$$
\partial^{\dot{\alpha} \alpha} j_{\alpha \dot{\alpha}}=0, \quad \partial^{\dot{\alpha} \alpha} S_{\alpha \beta \dot{\alpha}}=0, \quad \partial^{\dot{\alpha} \alpha} T_{\alpha \beta \dot{\alpha} \dot{\beta}}=0,
$$

as a consequence of (1.5). We point out that the energy-momentum $T^{a b}$ is automatically traceless and the four-component Majorana supersymmetry current is $\gamma$-traceless.

\footnotetext{
${ }^{5}$ The definition of the energy-momentum tensor given in section 5.7.3 of [9] contains an error.
} 
Here we do not list all the component fields of $L$. We only point out that the conserved current contained in $L$ is given by

$$
V_{\alpha \dot{\alpha}}:=\left[D_{\alpha}, \bar{D}_{\dot{\alpha}}\right] L \mid .
$$

In order to compute the conserved current contained in (4.2), it is necessary to make use of the explicit expression for the most general $\mathcal{N}=1$ conformal Killing supervector field, which is given, e.g., in $[9,36]$.

\section{$4.2 \mathcal{N}=2$ superconformal symmetry}

Consider an arbitrary $\mathcal{N}=2$ conformal Killing supervector field,

$$
\xi=\xi^{a} \partial_{a}+\xi_{i}^{\alpha} D_{\alpha}^{i}+\bar{\xi}_{\dot{\alpha}}^{i} \bar{D}_{i}^{\dot{\alpha}} .
$$

Its components are constrained according to (2.6). Let $J$ be the $\mathcal{N}=2$ conformal supercurrent. As discussed in [12], $J$ is a primary real scalar superfield of dimension +2 and obeys the conservation equation (1.6). We introduce the following real SU(2) triplet

$$
L^{i j}=\frac{\mathrm{i}}{8} \xi^{\dot{\alpha} \alpha}\left[D_{\alpha}^{(i}, \bar{D}_{\dot{\alpha}}^{j)}\right] J-\Lambda^{i j} J+\left(\xi^{\alpha(i} D_{\alpha}^{j)}+\bar{\xi}^{\dot{\alpha}(i} \bar{D}_{\dot{\alpha}}^{j)}\right) J .
$$

Here the real $\mathrm{SU}(2)$ triplet $\Lambda^{i j}$ is defined as

$$
\Lambda^{i j}=-\frac{\mathrm{i}}{32}\left[D_{\alpha}^{(i}, \bar{D}_{\dot{\alpha}}^{j)}\right] \xi^{\alpha \dot{\alpha}}
$$

and has the properties [12]

$$
D_{\alpha}^{(i} \Lambda^{j k)}=0 \quad \Longleftrightarrow \quad \bar{D}_{\dot{\alpha}}^{(i} \Lambda^{j k)}=0
$$

One can check that $L^{i j}$ is a linear multiplet,

$$
D_{\alpha}^{(i} L^{j k)}=\bar{D}_{\dot{\alpha}}^{(i} L^{j k)}=0
$$

and therefore it contains a conserved current.

The component content of $J$ and $L^{i j}$ is discussed, e.g., in [12].

\section{Superconformal theories in five and six dimensions}

The unique feature of five spacetime dimensions is that there is only one superconformal algebra [18], which is isomorphic to the exceptional superalgebra $F^{2}(4)$ and corresponds to the supersymmetry type $\mathcal{N}=1$ with eight supercharges. Our $5 \mathrm{D}$ superspace notation and conventions correspond to [38] with one exception. Instead of using Greek letters with a hat, such as $\hat{\alpha}, \hat{\beta}$, for the four-component spinor indices [38], here such indices will be denoted by ordinary Greek letters.

Any real supervector field $\xi$ on $5 \mathrm{D} \mathcal{N}=1$ Minkowski superspace $\mathbb{M}^{5 \mid 8}$ has the form

$$
\xi=\xi^{A} D_{A}=\xi^{a} \partial_{a}+\xi_{i}^{\alpha} D_{\alpha}^{i}, \quad i=1,2,
$$


where $\xi^{a}$ is real and $\xi_{i}^{\alpha}$ obeys the pseudo-Majorana condition defined in appendix A of [38]. Requiring $\xi$ to be conformal Killing, eq. (2.1), one obtains [16] (see also [39])

$$
\begin{aligned}
\xi_{\alpha}^{i} & =\frac{\mathrm{i}}{10} D^{\beta i} \xi_{\beta \alpha}, \\
D_{(\alpha}^{i} \xi_{\beta) \gamma} & =-\frac{1}{5} D^{\delta i} \xi_{\delta(\alpha} \varepsilon_{\beta) \gamma},
\end{aligned}
$$

where the traceless antisymmetric rank-two spinor $\xi_{\alpha \beta}$ is obtained from $\xi^{a}$ by the standard rule $\xi_{\alpha \beta}=\left(\Gamma_{c}\right)_{\alpha \beta} \xi^{c}$, with the $\Gamma$-matrices defined as in [38]. Eq. (5.2b) is the 5D superconformal Killing equation. One can deduce from (5.2) the following identities:

$$
D_{\alpha}^{(i} \xi_{\beta}^{j)}=\frac{1}{4} \varepsilon_{\alpha \beta} D^{\gamma(i} \xi_{\gamma}^{j)} \quad \Longrightarrow \quad D_{\alpha}^{(i} D_{\beta}^{j} \xi_{\gamma}^{k)}=0, \quad\left(\Gamma^{b}\right)_{\alpha \beta} D^{\alpha i} \xi_{i}^{\beta}=0
$$

The $\mathcal{N}=1$ and $\mathcal{N}=2$ supercurrents in five dimensions were introduced by Howe and Lindström [40]. The conformal supercurrent, $J$, is a primary real scalar superfield of dimension +3 , which obeys the conservation equation [39]

$$
D^{\alpha(i} D_{\alpha}^{j)} J=0 \quad \Longrightarrow \quad D_{\alpha}^{(i} D_{\beta}^{j} D_{\gamma}^{k)} J=0 .
$$

Given a conformal Killing supervector field $\xi^{A}$, we consider the following descendant of the supercurrent:

$$
L^{i j}=\frac{\mathrm{i}}{8} \xi^{\alpha \beta} D_{\alpha}^{(i} D_{\beta}^{j)} J-\xi^{\alpha(i} D_{\alpha}^{j)} J+\Lambda^{i j} J
$$

where $\Lambda^{i j}$ is defined by

$$
\Lambda^{i j}=\frac{1}{4} D_{\alpha}^{\left(i \xi^{j}\right) \alpha}
$$

and obeys the constraint

$$
D_{\alpha}^{(i} \Lambda^{j k)}=0 .
$$

Making use of the identities (5.2) and (5.3) and the conservation equation (5.4), one may check that $L^{i j}$ is a linear multiplet,

$$
D_{\alpha}^{(i} L^{j k)}=0
$$

and therefore it contains a conserved current.

The expressions (4.8) and (5.5) look very similar. This feature is not accidental and actually it follows from the fact that the $4 \mathrm{D} \mathcal{N}=2$ and $5 \mathrm{D} \mathcal{N}=1$ supersymmetries describe eight supercharges. Another case with eight supercharges is the $6 \mathrm{D} \mathcal{N}=(1,0)$ supersymmetry, to which the above 5D analysis extends almost without changes. The only difference between the $5 \mathrm{D}$ and $6 \mathrm{D}$ cases is that the $6 \mathrm{D} \mathcal{N}=(1,0)$ conformal supercurrent, $J$, is a primary real scalar superfield of dimension +4 , which obeys the conservation equation [41]

$$
D_{\alpha}^{(i} D_{\beta}^{j} D_{\gamma}^{k)} J=0
$$

which differs from the 5D conservation equation (5.4). However, the only property of the 5 D supercurrent, which was crucial in order to establish (5.8), was the relation on the right hand side of (5.4). The latter is the $5 \mathrm{D}$ counterpart of the $6 \mathrm{D}$ conservation equation (5.9). 


\section{Non-conformal supercurrents, curved backgrounds}

In this paper, we have presented the supersymmetric extensions of the construction $\left(T^{a b}, \xi^{a}\right) \rightarrow V^{a}$, where $T^{a b}$ is the conserved and traceless energy-momentum tensor, $\xi^{a}$ is an arbitrary conformal Killing vector field, and $V^{a}$ is the conserved current defined by (1.3). As is well known, the field-theoretic construction has a simple modification to the non-conformal case when $T^{a b}$ is no longer traceless,

$$
T^{a b}=T^{b a}, \quad \partial_{b} T^{a b}=0 .
$$

The vector field $V^{a}$ defined by (1.3) is still conserved provided $\xi^{a}$ is a Killing vector field,

$$
\partial_{a} \xi_{b}+\partial_{b} \xi_{a}=0
$$

This non-conformal construction also admits a supersymmetric generalisation. We will describe it only in the $4 \mathrm{D}$ case. To start with, we will briefly recall the structure of $\mathcal{N}=1$ and $\mathcal{N}=2$ non-conformal supercurrents.

A general non-conformal $\mathcal{N}=1$ supercurrent is naturally associated with the nonminimal off-shell formulation $[42,43]$ for $\mathcal{N}=1$ supergravity. The supercurrent conservation equation (see, e.g., [44]) is

$$
\bar{D}^{\dot{\alpha}} J_{\alpha \dot{\alpha}}=a \bar{D}^{2} \zeta_{\alpha}-b D_{\alpha} \bar{D}_{\dot{\beta}} \bar{\zeta}^{\dot{\beta}}, \quad D_{(\alpha} \zeta_{\beta)}=0
$$

with $a, b$ real parameters. Setting $\zeta_{\alpha}=D_{\alpha} Z$ leads to the supercurrent multiplet derived in [45] using a version of the superfield Noether procedure elaborated in [36].

An alternative form for the general $\mathcal{N}=1$ supercurrent, which is simply related to (6.3), was presented in [46]. It naturally follows from the classification of the linearised $\mathcal{N}=1$ supergravity actions given in [47] and is described by the conservation equation

$$
\begin{aligned}
\bar{D}^{\dot{\alpha}} J_{\alpha \dot{\alpha}} & =\chi_{\alpha}+\mathrm{i} \eta_{\alpha}+D_{\alpha} X, \\
\bar{D}_{\dot{\alpha}} \chi_{\alpha} & =\bar{D}_{\dot{\alpha}} \eta_{\alpha}=\bar{D}_{\dot{\alpha}} X=0, \quad D^{\alpha} \chi_{\alpha}-\bar{D}_{\dot{\alpha}} \bar{\chi}^{\dot{\alpha}}=D^{\alpha} \eta_{\alpha}-\bar{D}_{\dot{\alpha}} \bar{\eta}^{\dot{\alpha}}=0 .
\end{aligned}
$$

The chiral superfields $\chi_{\alpha}, \eta_{\alpha}$ and $X$ constitute the so-called multiplet of anomalies. In principle, one may always solve the constraints imposed on $\chi_{\alpha}, \eta_{\alpha}$ and $X$ in terms of unconstrained potentials as follows

$$
\chi_{\alpha}=-\frac{1}{4} \bar{D}^{2} D_{\alpha} V, \quad \eta_{\alpha}=-\frac{1}{4} \bar{D}^{2} D_{\alpha} U, \quad X=-\frac{1}{4} \bar{D}^{2} Z,
$$

where $V$ and $U$ are real. However, in some cases this is accompanied by the loss of locality (that is, some of the potentials are not well-defined local operators) and gauge invariance. This point of view was advocated in [48]. The supercurrent (6.4) with $\chi_{\alpha}=\eta_{\alpha}=0$ was introduced by Ferrara and Zumino [1], and it is associated with the old minimal formulation [49-51] for $\mathcal{N}=1$ supergravity. The supercurrent (6.4) with $\eta_{\alpha}=0$ and $X=0$ corresponds to the new minimal formulation [52] for $\mathcal{N}=1$ supergravity. Sometimes it is called the $R$-multiplet [48]. The supercurrent (6.4) with $\chi_{\alpha}=0$ and $X=0$ corresponds to the exotic minimal supergravity formulation [53], which is known only at the linearised level. 
This supercurrent is sometimes called the virial multiplet $[54,55]$. The supercurrent (6.4) with $\eta_{\alpha}=0$ is known as the $S$-multiplet [48]. It does not correspond to any irreducible supergravity theory, although it was argued [48] to be universal in the case of $\mathcal{N}=1$ Poincaré supersymmetry. ${ }^{6}$

Let us also reproduce a non-conformal deformation of the $\mathcal{N}=2$ supercurrent multiplet (1.6) that supports a large family of $\mathcal{N}=2$ supersymmetric field theories. The corresponding conservation equation $[56,57]$ is

$$
\frac{1}{4} D^{i j} J=w T^{i j}-g^{i j} Y
$$

where $T^{i j}$ and $Y$ are the trace multiplets constrained by

$$
\begin{aligned}
D_{\alpha}^{(i} T^{j k)}=\bar{D}_{\dot{\alpha}}^{(i} T^{j k)} & =0, & \overline{T^{i j}} & =T_{i j}, \\
\bar{D}_{\dot{\alpha}}^{i} Y & =0, & D^{i j} Y & =\bar{D}^{i j} \bar{Y},
\end{aligned}
$$

The right-hand side of (6.6a) involves two constant parameters, complex $w$ and real SU(2) triplet $g^{i j}$, which may be thought of as expectation values of the two conformal compensators in the off-shell formulations for $\mathcal{N}=2$ supergravity developed by de Wit, Philippe and Van Proeyen [59]. The supercurrent multiplet with $g^{i j}=0$ is equivalent to the one discovered originally by Sohnius [5].

In the remainder of this section, our analysis will be restricted to the $\mathcal{N}=1$ case and only the Ferrara-Zumino supercurrent [1] will be studied (all technical steps are analogous for the other supercurrents). The corresponding conservation equation is

$$
\bar{D}^{\dot{\alpha}} J_{\alpha \dot{\alpha}}=D_{\alpha} X, \quad \bar{D}_{\dot{\alpha}} X=0,
$$

with $X$ the chiral trace multiplet. ${ }^{7}$ If $X \neq 0$, the real scalar $L$ defined by (4.2) is no longer a linear superfield. Conservation equation (4.3) turns into

$$
\bar{D}^{2} L=2 \mathrm{i} \xi X=2 \mathrm{i}\left(\xi^{a} \partial_{a}+\xi^{\alpha} D_{\alpha}\right) X
$$

Here the right-hand side is chiral, because $\xi X$ is the variation of the chiral superfield $X$ generated by the conformal Killing supervector field $\xi=\xi^{A} D_{A}$. If $\xi$ is a Killing supervector field, then it obeys the additional constraint [9]

$$
D_{\alpha} \xi^{\alpha}=\bar{D}_{\dot{\alpha}} \bar{\xi}^{\dot{\alpha}}=0 \quad \Longrightarrow \quad \partial_{a} \xi^{a}=0
$$

In the case that $\xi$ is Killing, the relation (6.8) is equivalent to

$$
\bar{D}^{2} L=2 \mathrm{i} \xi X=2 \mathrm{i}\left\{\partial_{a}\left(\xi^{a} X\right)-D_{\alpha}\left(\xi^{\alpha} X\right)\right\} .
$$

\footnotetext{
${ }^{6}$ The $S$-multiplet does not exist in the case of $\mathcal{N}=1$ anti-de Sitter supersymmetry [56], for which the Ferrara-Zumino supercurrent is universal.

${ }^{7}$ Since $D^{2} X-\bar{D}^{2} \bar{X}=-2 \mathrm{i} \partial_{\alpha \dot{\alpha}} J^{\dot{\alpha} \alpha}$, the chiral trace $X$ in (6.7) is in fact an example of the three-form multiplet $[44,58]$.
} 
Since $\bar{D}^{(\dot{\alpha}} \xi^{\dot{\beta}) \beta}=0$, eq. (2.6b), we can represent

$$
\xi_{\alpha \dot{\alpha}}=-2 \mathrm{i} \bar{D}_{\dot{\alpha}} \Upsilon_{\alpha} \quad \Longrightarrow \quad \xi_{\alpha}=-\frac{1}{4} \bar{D}^{2} \Upsilon_{\alpha},
$$

for some spinor $\Upsilon_{\alpha}$. Making use of this representation, eq. (6.10) may be rewritten in the form

$$
\bar{D}^{2} \tilde{L}=0, \quad \tilde{L}:=L+\frac{\mathrm{i}}{2}\left\{D^{\alpha}\left(\Upsilon_{\alpha} X\right)-\bar{D}_{\dot{\alpha}}\left(\bar{\Upsilon}^{\dot{\alpha}} \bar{X}\right)\right\} .
$$

We conclude that $\tilde{L}$ contains a conserved current.

So far, our discussion in this paper has been restricted to theories in flat superspace. However, practically all considerations and conclusions may be extended to supersymmetric field theories defined on curved superspace backgrounds with symmetries. As an example, let us consider a curved superspace background $\mathcal{M}^{4 \mid 4}$ of the $4 \mathrm{D} \mathcal{N}=1$ old minimal supergravity. ${ }^{8}$ Let $J_{\alpha \dot{\alpha}}$ be the conformal supercurrent,

$$
\mathcal{D}^{\alpha} J_{\alpha \dot{\alpha}}=0 \quad \Longrightarrow \quad\left(\mathcal{D}^{2}-6 \bar{R}\right) J_{\alpha \dot{\alpha}}=0
$$

Let $\xi=\xi^{A} E_{A}$ be a conformal Killing supervector field of $\mathcal{M}^{4 \mid 4}$. As demonstrated in section 6.4 of [9], its explicit form is

$$
\xi^{A}=\left(\xi^{a}, \xi^{\alpha}, \bar{\xi}_{\dot{\alpha}}\right)=\left(\xi^{a},-\frac{\mathrm{i}}{8} \overline{\mathcal{D}}_{\dot{\beta}} \xi^{\dot{\beta} \alpha},-\frac{\mathrm{i}}{8} \mathcal{D}^{\beta} \xi_{\beta \dot{\alpha}}\right)
$$

where the vector component $\xi_{\alpha \dot{\alpha}}$ is real and obeys the equation

$$
\mathcal{D}_{(\alpha} \xi_{\beta) \dot{\beta}}=0 \quad \Longrightarrow \quad\left(\mathcal{D}^{2}+2 \bar{R}\right) \xi_{\alpha \dot{\alpha}}=0
$$

Then the real scalar $L:=-\frac{1}{2} \xi^{\alpha \dot{\alpha}} J_{\alpha \dot{\alpha}}$ is a conserved current multiplet,

$$
\left(\mathcal{D}^{2}-4 \bar{R}\right) L=0
$$

\section{Acknowledgments}

The work of SMK and ST is supported in part by the Australian Research Council (ARC) Discovery Project DP140103925.

\section{A Conformal symmetries of curved superspace}

The material in this section is taken almost verbatim from [60].

Let $\mathcal{M}^{d \mid \delta}$ be a curved superspace, with $d$ spacetime and $\delta$ fermionic dimensions, chosen to describe a given supergravity theory. We denote by $z^{M}=\left(x^{m}, \theta^{\hat{\mu}}\right)$ the local coordinates for $\mathcal{M}^{d \mid \delta}$. Without loss of generality, we assume that the zero section of $\mathcal{M}^{d \mid \delta}$ defined by $\theta^{\hat{\mu}}=0$ corresponds to the spacetime manifold $\mathcal{M}^{d}$.

\footnotetext{
${ }^{8}$ Our supergravity conventions follow [9] and slightly differ from those used in [19].
} 
The differential geometry of curved superspace $\mathcal{M}^{d \mid \delta}$ may be realised in terms of covariant derivatives of the form

$$
\mathcal{D}_{A}=\left(\mathcal{D}_{a}, \mathcal{D}_{\hat{\alpha}}\right)=E_{A}+\Omega_{A}+\Phi_{A} .
$$

Here $E_{A}=E_{A}{ }^{M}(z) \partial / \partial z^{M}$ denotes the inverse superspace vielbein, $\Omega_{A}=\frac{1}{2} \Omega_{A}{ }^{b c}(z) M_{b c}$ is the Lorentz connection, and $\Phi=\Phi_{A}{ }^{I}(z) J_{I}$ the $R$-symmetry connection. ${ }^{9}$ The index $\hat{\alpha}$ of the fermionic operator $\mathcal{D}_{\hat{\alpha}}$ is, in general, composite; it is comprised of a spinor index $\alpha$ and an $R$-symmetry index.

The covariant derivatives obey the (anti-)commutation relations of the form

$$
\left[\mathcal{D}_{A}, \mathcal{D}_{B}\right\}=\mathcal{T}_{A B}{ }^{C} \mathcal{D}_{C}+\frac{1}{2} \mathcal{R}_{A B}{ }^{c d} M_{c d}+\mathcal{R}_{A B}{ }^{I} J_{I}
$$

where $\mathcal{T}_{A B}{ }^{C}(z)$ is the torsion tensor, $\mathcal{R}_{A B}{ }^{c d}(z)$ and $\mathcal{R}_{A B}{ }^{I}(z)$ are the Lorentz and $R$ symmetry curvature tensors, respectively. In order to describe conformal supergravity, the superspace torsion $\mathcal{T}_{A B}{ }^{C}$ has to obey certain algebraic constraints.

The supergravity gauge group includes a subgroup generated by local transformations

$$
\delta_{\mathcal{K}} \mathcal{D}_{A}=\left[\mathcal{K}, \mathcal{D}_{A}\right], \quad \mathcal{K}:=\xi^{B}(z) \mathcal{D}_{B}+\frac{1}{2} K^{b c}(z) M_{b c}+K^{I}(z) J_{I},
$$

where the gauge parameters $\xi^{B}, K^{b c}=-K^{c b}$ and $K^{I}$ obey standard reality conditions but are otherwise arbitrary.

In order to describe conformal supergravity, the constraints imposed on the superspace torsion should be invariant under super-Weyl transformations [61] of the form

$$
\delta_{\sigma} \mathcal{D}_{a}=\sigma \mathcal{D}_{a}+\cdots, \quad \delta_{\sigma} \mathcal{D}_{\hat{\alpha}}=\frac{1}{2} \sigma \mathcal{D}_{\hat{\alpha}}+\cdots,
$$

where the scale parameter $\sigma$ is an arbitrary real superfield. The ellipsis in the expression for $\delta_{\sigma} \mathcal{D}_{a}$ includes, in general, a linear combination of the spinor covariant derivatives $\mathcal{D}_{\hat{\beta}}$ and the structure group generators $M_{c d}$ and $J_{K}$. The ellipsis in $\delta_{\sigma} \mathcal{D}_{\hat{\alpha}}$ stands for a linear combination of the generators of the structure group. Consider the superspace vielbein $E^{A}=\mathrm{d} z^{M} E_{M}{ }^{A}(z)$ to which $E_{A}$ is dual. The specific feature of the super-Weyl transformation is that the vector one-form $E^{a}$ transforms homogeneously,

$$
\delta_{\sigma} E^{a}=-\sigma E^{a}
$$

This implies that every super-Weyl transformation at most scales the superspace interval defined by $\mathrm{d} s^{2}:=\eta_{a b} E^{a} E^{b}$. The Lorentz and $R$-symmetry transformations preserve the interval.

Let us now fix a background superspace. A supervector field $\xi=\xi^{B} E_{B}$ on $\left(\mathcal{M}^{d \mid \delta}, \mathcal{D}\right)$ is called conformal Killing if

$$
\left(\delta_{\mathcal{K}}+\delta_{\sigma}\right) \mathcal{D}_{A}=0,
$$

\footnotetext{
${ }^{9}$ The superspace structure group, $\operatorname{Spin}(d-1,1) \times G_{R}$, is a subgroup of the isometry group of Minkowski superspace $\mathbb{M}^{d \mid \delta}$. This subgroup is the isotropy group of the origin in $\mathbb{M}^{d \mid \delta}$.
} 
for some Lorentz $K^{b c}, R$-symmetry $K^{I}$ and super-Weyl $\sigma$ parameters. For any dimension $d \leq 6$ and any conformal supergravity with up to eight supercharges, the following properties hold: (i) all parameters $K^{b c}, K^{I}$ and $\sigma$ are uniquely determined in terms of $\xi^{B}$, which allows us to write $K^{b c}=K^{b c}[\xi], K^{I}=K^{I}[\xi]$ and $\sigma=\sigma[\xi]$; (ii) the spinor component $\xi^{\hat{\beta}}$ is uniquely determined in terms of $\xi^{b}$; (iii) the vector component $\xi^{b}$ obeys a superconformal Killing equation, which contains all the information about the conformal Killing vector field and, in particular, implies the ordinary conformal Killing equation

$$
\mathcal{D}_{a} \xi_{b}+\mathcal{D}_{b} \xi_{a}=\frac{2}{d} \eta_{a b} \mathcal{D}_{c} \xi^{c}
$$

Unlike (A.7), the explicit form of the superconformal Killing equation depends on the spacetime dimension and supersymmetry type chosen. For instance, in the case of 4D $\mathcal{N}=1$ supergravity this equation $[9]$ is given by $(6.15)$.

Open Access. This article is distributed under the terms of the Creative Commons Attribution License (CC-BY 4.0), which permits any use, distribution and reproduction in any medium, provided the original author(s) and source are credited.

\section{References}

[1] S. Ferrara and B. Zumino, Transformation properties of the supercurrent, Nucl. Phys. B 87 (1975) 207 [inSPIRE].

[2] S. Ferrara, J. Wess and B. Zumino, Supergauge multiplets and superfields, Phys. Lett. B 51 (1974) 239 [INSPIRE].

[3] M.F. Sohnius, The multiplet of currents for $N=2$ extended supersymmetry, Phys. Lett. B 81 (1979) 8 [INSPIRE].

[4] P. Breitenlohner and M.F. Sohnius, Superfields, auxiliary fields and tensor calculus for $N=2$ extended supergravity, Nucl. Phys. B 165 (1980) 483 [INSPIRE].

[5] M.F. Sohnius, The conformal group in superspace, in Quantum Theory and the Structures of Time and Space, vol. 2, L. Castell, M. Drieschner and C.F. von Weizsäcker eds., Carl Hanser Verlag, München Germany (1977), pg. 241 [INSPIRE].

[6] W. Lang, Construction of the minimal superspace translation tensor and the derivation of the supercurrent, Nucl. Phys. B 179 (1981) 106 [inSPIRE].

[7] L. Bonora, P. Pasti and M. Tonin, Cohomologies and anomalies in supersymmetric theories, Nucl. Phys. B 252 (1985) 458 [inSPIRE].

[8] K.-I. Shizuya, Supercurrents and superconformal symmetry, Phys. Rev. D 35 (1987) 1848 [INSPIRE].

[9] I.L. Buchbinder and S.M. Kuzenko, Ideas and methods of supersymmetry and supergravity or a walk through superspace, revised edition, IOP, Bristol U.K. (1998) [INSPIRE].

[10] P.S. Howe and G.G. Hartwell, A superspace survey, Class. Quant. Grav. 12 (1995) 1823 [INSPIRE].

[11] J.-H. Park, Superconformal symmetry and correlation functions, Nucl. Phys. B 559 (1999) 455 [hep-th/9903230] [INSPIRE]. 
[12] S.M. Kuzenko and S. Theisen, Correlation functions of conserved currents in $N=2$ superconformal theory, Class. Quant. Grav. 17 (2000) 665 [hep-th/9907107] [INSPIRE].

[13] C. Grojean and J. Mourad, Superconformal $6 D(2,0)$ theories in superspace, Class. Quant. Grav. 15 (1998) 3397 [hep-th/9807055] [INSPIRE].

[14] J.-H. Park, Superconformal symmetry in six-dimensions and its reduction to four-dimensions, Nucl. Phys. B 539 (1999) 599 [hep-th/9807186] [InSPIRE].

[15] J.-H. Park, Superconformal symmetry in three-dimensions, J. Math. Phys. 41 (2000) 7129 [hep-th/9910199] [INSPIRE].

[16] S.M. Kuzenko, On compactified harmonic/projective superspace, $5 D$ superconformal theories and all that, Nucl. Phys. B 745 (2006) 176 [hep-th/0601177] [INSPIRE].

[17] S.M. Kuzenko, J.-H. Park, G. Tartaglino-Mazzucchelli and R. Unge, Off-shell superconformal nonlinear $\sigma$-models in three dimensions, JHEP 01 (2011) 146 [arXiv:1011.5727] [INSPIRE].

[18] W. Nahm, Supersymmetries and their representations, Nucl. Phys. B 135 (1978) 149 [INSPIRE].

[19] J. Wess and J. Bagger, Supersymmetry and supergravity, Princeton University Press, Princeton U.S.A. (1992) [INSPIRE].

[20] D.V. Volkov and V.P. Akulov, Is the neutrino a Goldstone particle?, Phys. Lett. B 46 (1973) 109 [INSPIRE].

[21] V.P. Akulov and D.V. Volkov, Goldstone fields with spin 1/2, Theor. Math. Phys. 18 (1974) 28 [Teor. Mat. Fiz. 18 (1974) 39] [InSPIRE].

[22] P.S. Howe and U. Lindström, Notes on super Killing tensors, JHEP 03 (2016) 078 [arXiv: 1511.04575] [INSPIRE].

[23] S.M. Kuzenko, J. Novak and G. Tartaglino-Mazzucchelli, $N=6$ superconformal gravity in three dimensions from superspace, JHEP 01 (2014) 121 [arXiv:1308.5552] [INSPIRE].

[24] D. Butter, S.M. Kuzenko, J. Novak and G. Tartaglino-Mazzucchelli, Conformal supergravity in three dimensions: new off-shell formulation, JHEP 09 (2013) 072 [arXiv:1305.3132] [INSPIRE].

[25] E.I. Buchbinder, S.M. Kuzenko and I.B. Samsonov, Superconformal field theory in three dimensions: correlation functions of conserved currents, JHEP 06 (2015) 138 [arXiv: 1503.04961] [INSPIRE].

[26] E.I. Buchbinder, S.M. Kuzenko and I.B. Samsonov, Implications of $N=4$ superconformal symmetry in three spacetime dimensions, JHEP 08 (2015) 125 [arXiv:1507.00221] [INSPIRE].

[27] T.T. Dumitrescu and N. Seiberg, Supercurrents and brane currents in diverse dimensions, JHEP 07 (2011) 095 [arXiv:1106.0031] [INSPIRE].

[28] S.M. Kuzenko and G. Tartaglino-Mazzucchelli, Three-dimensional $N=2$ (AdS) supergravity and associated supercurrents, JHEP 12 (2011) 052 [arXiv:1109.0496] [INSPIRE].

[29] W. Siegel, Unextended superfields in extended supersymmetry, Nucl. Phys. B 156 (1979) 135 [INSPIRE].

[30] N.J. Hitchin, A. Karlhede, U. Lindström and M. Roček, Hyper-Kähler metrics and supersymmetry, Commun. Math. Phys. 108 (1987) 535 [INSPIRE]. 
[31] B.M. Zupnik and D.G. Pak, Superfield formulation of the simplest three-dimensional gauge theories and conformal supergravities, Theor. Math. Phys. 77 (1988) 1070 [Teor. Mat. Fiz. 77 (1988) 97] [INSPIRE].

[32] B.M. Zupnik and D.V. Khetselius, Three-dimensional extended supersymmetry in the harmonic superspace (in Russian), Sov. J. Nucl. Phys. 47 (1988) 730 [Yad. Fiz. 47 (1988) 1147] [INSPIRE].

[33] S.M. Kuzenko, U. Lindström and G. Tartaglino-Mazzucchelli, Off-shell supergravity-matter couplings in three dimensions, JHEP 03 (2011) 120 [arXiv:1101.4013] [INSPIRE].

[34] U. Gran, J. Greitz, P.S. Howe and B.E.W. Nilsson, Topologically gauged superconformal Chern-Simons matter theories, JHEP 12 (2012) 046 [arXiv:1204.2521] [INSPIRE].

[35] S.M. Kuzenko and J. Novak, Supergravity-matter actions in three dimensions and Chern-Simons terms, JHEP 05 (2014) 093 [arXiv:1401.2307] [INSPIRE].

[36] H. Osborn, $N=1$ superconformal symmetry in four-dimensional quantum field theory, Annals Phys. 272 (1999) 243 [hep-th/9808041] [INSPIRE].

[37] P.S. Howe, K.S. Stelle and P.K. Townsend, Supercurrents, Nucl. Phys. B 192 (1981) 332 [INSPIRE].

[38] S.M. Kuzenko and W.D. Linch, III, On five-dimensional superspaces, JHEP 02 (2006) 038 [hep-th/0507176] [INSPIRE].

[39] S.M. Kuzenko, J. Novak and G. Tartaglino-Mazzucchelli, Symmetries of curved superspace in five dimensions, JHEP 10 (2014) 175 [arXiv:1406.0727] [INSPIRE].

[40] P.S. Howe and U. Lindström, The supercurrent in five-dimensions, Phys. Lett. B 103 (1981) 422 [INSPIRE].

[41] P.S. Howe, G. Sierra and P.K. Townsend, Supersymmetry in six-dimensions, Nucl. Phys. B 221 (1983) 331 [InSPIRE].

[42] P. Breitenlohner, Some invariant Lagrangians for local supersymmetry, Nucl. Phys. B 124 (1977) 500 [inSPIRE].

[43] W. Siegel and S.J. Gates, Jr., Superfield supergravity, Nucl. Phys. B 147 (1979) 77 [INSPIRE].

[44] S.J. Gates, M.T. Grisaru, M. Roček and W. Siegel, Superspace or one thousand and one lessons in supersymmetry, Front. Phys. 58 (1983) 1 [hep-th/0108200] [INSPIRE].

[45] M. Magro, I. Sachs and S. Wolf, Superfield Noether procedure, Annals Phys. 298 (2002) 123 [hep-th/0110131] [INSPIRE].

[46] S.M. Kuzenko, Variant supercurrent multiplets, JHEP 04 (2010) 022 [arXiv:1002.4932] [INSPIRE].

[47] S.J. Gates Jr., S.M. Kuzenko and J. Phillips, The off-shell $(3 / 2,2)$ supermultiplets revisited, Phys. Lett. B 576 (2003) 97 [hep-th/0306288] [INSPIRE].

[48] Z. Komargodski and N. Seiberg, Comments on supercurrent multiplets, supersymmetric field theories and supergravity, JHEP 07 (2010) 017 [arXiv: 1002.2228] [INSPIRE].

[49] J. Wess and B. Zumino, Superfield Lagrangian for supergravity, Phys. Lett. B 74 (1978) 51 [INSPIRE]. 
[50] K.S. Stelle and P.C. West, Minimal auxiliary fields for supergravity, Phys. Lett. B 74 (1978) 330 [InSPIRE].

[51] S. Ferrara and P. van Nieuwenhuizen, The auxiliary fields of supergravity, Phys. Lett. B 74 (1978) 333 [INSPIRE].

[52] M.F. Sohnius and P.C. West, An alternative minimal off-shell version of $N=1$ supergravity, Phys. Lett. B 105 (1981) 353 [inSPIRE].

[53] I.L. Buchbinder, S.J. Gates, Jr., W.D. Linch, III and J. Phillips, New $4 D, N=1$ superfield theory: model of free massive superspin $3 / 2$ multiplet, Phys. Lett. B 535 (2002) 280 [hep-th/0201096] [INSPIRE].

[54] Y. Nakayama, Supercurrent, supervirial and superimprovement, Phys. Rev. D 87 (2013) 085005 [arXiv:1208.4726] [INSPIRE].

[55] Y. Nakayama, Imaginary supergravity or virial supergravity?, Nucl. Phys. B 892 (2015) 288 [arXiv: 1411.1057] [INSPIRE].

[56] D. Butter and S.M. Kuzenko, $N=2$ AdS supergravity and supercurrents, JHEP 07 (2011) 081 [arXiv: 1104.2153] [INSPIRE].

[57] D. Butter and S.M. Kuzenko, $N=2$ supergravity and supercurrents, JHEP 12 (2010) 080 [arXiv: 1011.0339] [INSPIRE].

[58] S.J. Gates, Jr., Super p-form gauge superfields, Nucl. Phys. B 184 (1981) 381 [INSPIRE].

[59] B. de Wit, R. Philippe and A. Van Proeyen, The improved tensor multiplet in $N=2$ supergravity, Nucl. Phys. B 219 (1983) 143 [INSPIRE].

[60] S.M. Kuzenko, Supersymmetric spacetimes from curved superspace, PoS (CORFU2014) 140 [arXiv: 1504.08114] [INSPIRE].

[61] P.S. Howe and R.W. Tucker, Scale invariance in superspace, Phys. Lett. B 80 (1978) 138 [INSPIRE]. 